\section{Software matemático para comprobar la resolución de ejercicios en bachillerato general unificado en Ecuador}

Mathematical software to check the resolution of exercises in unified general high school in Ecuador

\section{Chila Ortiz Hernan* \\ Hernández Martínez Jeimy* \\ Chávez Ruano Lucia* \\ Clavijo Robinson Isabel*}

\section{RESUMEN}

El uso de software matemáticos en los ciclos de aprendizaje de la asignatura Matemática actualmente tiene su crecimiento en función de los distintos dispositivos móviles que abren las puertas a aplicaciones educativas para fortalecer aprendizajes significativos. Este estudio utiliza la investigación-acción con un enfoque cualitativo y cuantitativo, apoyado en los siguientes métodos: hipotético - deductivo y dialéctico - crítico. Como consecuencia del vínculo con la sociedad y la investigación en la Unidad de Educación Fiscal "Margarita Cortes" de la provincia de Esmeraldas-Ecuador, como muestra participaron 245 alumnos y 10 profesores de matemáticas del nivel de Bachillerato General Unificado; El resultado de la prueba $T$ de seriesrelacionadas (pretest y postest) es $0.000<0.050$; se afirma la existencia de una

\footnotetext{
* Master en Docencia y Desarrollo del Currículo - Docente de la Universidad Técnica Luis Vargas Torres - Facultad de la Pedagogía Email: hernan.chila@utelvt.edu.ec, https://orcid.org/0000-0002- 2855-1942

* Magister en Ciencias de la Educación- Docente de la Universidad Técnica Luis Vargas Torres - Facultad de la Pedagogía Email: jeimy.hernandez@utelvt.edu.ec, https://orcid.org/ 0000-000 I-6349-6603

* Master en Desarrollo del Pensamiento y la Educación- Docente de la Universidad Técnica Luis Vargas Torres - Facultad de la Pedagogía Email: lucia.chavez@utelvt.edu.ec, https://orcid.org/ 0000-000I-6858-3845

* Master Universitario en Atención en Necesidades Educativas Especiales en Educación Infantil y Primaria - Docente de la Universidad Técnica Luis Vargas Torres - Facultad de la Pedagogía isabel.clavijo.robinzon@utelvt.edu.ec, https://orcid.org/ 0000-0002-91760384
}

\section{REVISTATECNOLÓGICA ciencia y educación Edwards Deming}

ISSN: 2600-5867

\section{Atribución/Reconocimiento-NoCo} mercial- Compartirlgual 4.0 Licencia Pública Internacional — CC

\section{BY-NC-SA 4.0}

https://creativecommons.org/licenses /by-nc- sa/4.0/legalcode.es

Editado por: Tecnológico Superior Corporativo Edwards Deming Enero - Marzo Vol. 6 - I - 2022 https://revista-edwardsdeming.com/index.php/es e-ISSN: 2576-097I

Recibido: 22 julio 202I

Aprobado: 16 septiembre, 2021

Pag 64 - 
relación significativa entre los resultados del pretest y postest; demostrando que el uso de software matemático en la fase de aplicación del ciclo de aprendizaje y favorece la autonomía de los estudiantes en la verificación de la solución de ejercicios matemáticos. Se concluye que los softwares matemáticos que contribuyen a la verificación de la solución de ejercicios matemáticos, entre otros, son: GeoGebra; PHOTOMATH; MATHWAY; MAXT 9; SYMBOLAB; MATRIXAPP, que innova la estrategia docente de los profesores de matemáticas del Bachillerato General Unificado.

Palabras clave: software matemático; comprobación; solución de ejercicios

\section{ABSTRACT}

He uses of mathematical software in the learning cycles of the Mathematics subject is currently growing as a function of the different mobile devices that open the doors to educational applications to strengthen meaningful learning. This study uses action research with a qualitative and quantitative approach, supported by the following methods: hypothetical - deductive and dialectical - critical. As a consequence of the link with society and the research in the Fiscal Education Unit "Margarita Cortes" of the province of Esmeraldas-Ecuador, as a sample, 245 students and 10 mathematics teachers of the Unified General Baccalaureate level participated; The result of the T-test of related series (pretest and posttest) is $0.000<0.050$; the existence of a significant relationship between the pretest and posttest results is affirmed; demonstrating that the use of mathematical software in the application phase of the learning cycle favors the autonomy of students in verifying the solution of mathematical exercises. It is concluded that the mathematical softwares that contribute to the verification of the solution of mathematical exercises, among others, are: GeoGebra; PHOTOMATH; MATHWAY; MAXT 9; SYMBOLAB; MATRIXAPP, which innovates the teaching strategy of the mathematics teachers of the Unified General Baccalaureate.

Keywords: mathematical software; verification; exercise solution

\section{INTRODUCCIÓN}

La matemática es una disciplina que facilita el desarrollo del pensamiento matemático y el lenguaje universal, contribuyendo a la construcción del conocimiento; razón suficiente para que los docentes realicen una enseñanza eficiente y los estudiantes alcancen buenos desempeños académicos apoyados por el uso del software matemático que aporta a 
este fin. La comprobación de solución de problemas aritméticos y algebraicos con el uso de software matemático innova la mediación que desde la metodología se favorece la integración del conocimiento del área, el conocimiento del software, la coherencia didáctica que se precisa en la tarea, motiva la relación dinámica y enriquecedora de sabres lo que produce soluciones de calidad situadas en aprendizajes significativos.

Frente a los múltiples y diversos problemas en la enseñanza - aprendizaje de la matemática, se requieren nuevos enfoques tecnológicos para abordarlos. A su vez, es factor de superación de la baja autonomía en el estudiante de aquel déficit que resta las posibilidades de encontrar las soluciones a ejercicios que contextualizados en la realidad son verdaderos problemas, que en esta investigación fue expresado como "limitaciones en el conocimiento de recursos didácticos - tecnológicos para realizar la comprobación de la solución de ejercicios mediante el uso de software matemático".

Actualmente, el uso de software matemático o las aplicaciones matemáticas involucradas en el sistema educativo permiten perfeccionar los procesos de enseñanza y aprendizaje en esta área del conocimiento. Los softwares, facilitan la mediación de los conceptos matemáticos a través de vistas de resultados de soluciones de ejercicios, visualizaciones muy dinámicas de los lugares geométricos del desarrollo de procesos algorítmicos, este enfoque pedagógico conlleva la aplicación de estrategias metodológicas que discurren por cada una las fases del ciclo de aprendizaje de Experiencia, Reflexión, Conceptualización (construcción del conocimiento) y Aplicación (ERCA).

La trascendencia del software implica investigación que se expresa en la consolidación integral producto de la relación parte-todo que permite valorar cómo y cuánto los dominios que el estudiante alcanza juegan para apropiarse de nuevos conceptos y sus operaciones. Por consiguiente, con ello se alcanzan nuevos estadios del desarrollo. De ahí, la impronta que aportan los softwares matemáticos con sus códigos e interfaces para vincular a los estudiantes con soluciones creativas de cálculos cada vez más complejos, Se asume entonces desde esta investigación que el uso de los dispositivos tecnológicos no pueden faltar para una mediación renovada del aprendizaje.

La presente investigación aporta desde el proyecto de vinculación titulado La FACPED impulsando el desarrollo educativo, la cultura, los saberes ancestrales y la recreación en la provincia de Esmeraldas, una instrumentación de capacitación que viabiliza la relación universidad-comunidad para llevar procesos tecnológicos que se desarrollan y apropian en la académica y cuyo fin es superar el aprendizaje matemático de los estudiantes del bachillerato de los planteles educativas de la provincia.

Una forma de acercarse a lo planteado en el párrafo anterior, lo precisan Torregrosa, G. et al., (20l0)., cuando señalan que "las conceptualizaciones sobre la comprobación matemática, requieren un ambiente virtual de aprendizaje y sus influencias en la solución de problemas" (p.380)., lo virtual se lo puede entender en relación espacio web y espacio del aparato tecnológico, en ese contexto el objetivo es promover el desarrollo de demostraciones en los estudiantes de Educación Secundaria en los espacios de un aparato móvil o laptop. 
Instrumentar operaciones en los aparatos tecnológicos en el proceso investigativo conllevó la aplicación de métodos inductivos para determinar las percepciones de los profesores involucrados, un acercamiento a esa realidad refleja que subsisten concepciones duales. Es decir, operan sin y con tecnología en la mediación del aprendizaje. Se corrobora que la influencia del uso de software matemáticos sobre la comprobación de solución de ejercicios ayuda a establecer la diferencia entre comprobar y generalizar.

En la investigación de Fernández, I., Riveros, V., \& Montiel, G. (2017), se sostiene que "el software educativo, ha conquistado el desarrollo de las sociedades y en el ámbito educativo cumple un significativo papel la enseñanza - aprendizaje, como también trascendencia en la comunicación e información" (p.II).

Se planteó como objetivo de la investigación: ejecutar una capacitación en software educativo, software matemático y tipos de aplicaciones tecnológicas (App) para encontrar soluciones a ejercicios matemáticos. Proceso que permitió identificar los softwares matemáticos para comprobar la resolución de ejercicios en el Bachillerato General Unificado en la fase de aplicación del ciclo de aprendizaje ERCA. Según Fernández, Izquierdo y Lima, (2000). "El uso de software en la enseñanza de la matemática admite en los estudiantes el desarrollo de las operaciones intelectuales y facilita la construcción de su propio conocimiento" (p.I). Para estos investigadores, el software matemático aporta al perfeccionamiento de habilidades y destrezas integrando al trabajo intelectual del estudiante.

En esta circunstancia anotada, con el uso del software educativo la mediación se proyecta a suministrar a los estudiantes procesos para interiorizar la información del contenido matemático, que se transmite y divulga en los textos y redes, pero que requieren de apropiación de herramientas para entender su significado desde el software mediador de experiencias de construcción de los aprendizajes.

En relación con el qué instrumentalizar para resolver un ejercicio matemático contextualizado, Ángel y Bautista, (200I); Guedez, (2005); Oteiza y Silva, (2001) ya aportan significativamente criterios que precisan que con el uso apropiado de las herramientas tecnológicas los estudiantes, apoyados por el docente, pueden efectuar acciones que les encauzan a hipotetizar, examinar, experimentar y extraer conclusiones a partir de involucrarse en sistemas de simulación y modelación.

Por consiguiente, asumimos que efectivamente el uso adecuado del software matemático, ayuda al profesor-mediador a convertirse en un diseñador de situaciones de aprendizaje para desarrollar en el estudiante destrezas de autoaprendizaje que consolida la independencia facilitando la interacción dialógica entre estudiante - profesor que rompe con la pedagogía tradicional monogal. Con el empleo del software matemático, los maestros pueden adecuar las estrategias metodologías para integrar al sujeto que aprende a los procesos de apropiación de herramientas con las que construyen sus conocimientos a partir de resolver ejercicios situados en contextos.

La adquisición de las destrezas y competencias de la matemática permite a los individuos la interacción con una alta fluidez argumental del fenómeno que se expresa en el espacio 
(pantalla) del instrumento tecnológico móvil o laptop. Los entes matemáticos numéricos o algebraicos surgen de las actividades cotidianas simples y compleja, operar en esos contextos conlleva ejecutar acciones lógicas de razonamiento y el problema como tal tiene un fin en la tarea, esa necesidad que surge por la resolución que es interna es la motivación lo que conduce a tomar las mejores decisiones, facilitando, además, la comprensión de las representaciones numéricas, algebraicas, gráficas y estadísticas.

La comprobación de solución de ejercicios matemáticos es una actividad importante que permite validar los indicadores que conlleva la segunda actividad investigativa. Por tanto, es el eje principal de esta investigación. Con la comprobación se valora las operaciones intelectuales de análisis y razonamiento, adquiridas por los estudiantes. Se afirma que el currículo, la didáctica y los softwares educativos fortalecen los procesos de enseñanzaaprendizaje para que los estudiantes desarrollen competencias para hallar los resultados de ejercicios de manera eficaz y consciente.

El ciclo de aprendizaje ERCA, según Rodríguez, (2017); “es una organización de acciones de aprendizaje que el docente propone, en función de cómo cree, que el estudiante debe aprender"., (p.37). En la definición que se propone queda claro que existe un proceso de ejecución de acciones pedagógicas orientadas desde la mediación a la construcción del conocimiento, en su secuencia lógica se revelan fases; la organización propicia una buena percepción del proceso de enseñanza que media el aprendizaje significativo; cuenta con cuatro fases distintas: Experiencia, Reflexión, Conceptualización y Aplicación; los mismos que, suministran verdaderos talleres, capacitaciones y orientaciones y hace de los profesores gestores de implementación del ciclo del aprendizaje.

La fase de aplicación es la última del ciclo de aprendizaje ERCA, es el espacio donde los estudiantes al interactuar consolidan la resolución de ejercicios matemáticos, demostrando dominio de saberes conocidos o aplicados a nuevos conocimientos. Es una etapa de perfeccionamiento de capacidades con actividades concretas situadas en ejercicios prácticos de conocimientos adquiridos. Va en la vía de "aplicar lo aprendido", es un suceso experiencial que integra la segunda etapa de conceptualización.

Al momento de que el estudiante realiza la fase de aplicación del ciclo ERCA se afirma el conocimiento. Por lo tanto, en la disciplina de matemática logra la comprobación de la resolución de ejercicios afirmando la construcción del conocimiento manera activa. En esta etapa el estudiante reflexiona y valora la validez de la solución del ejercicio, aunque se haya realizado con diferente proceso.

Según Blanco, L., Cárdenas, J., Caballero, A. (2015). consideraban que "en la fase de aplicación del ciclo de aprendizaje ERCA permite a los estudiantes comprender asertivamente los ejercicios planteados y alcancen el conocimiento conscientes y buen uso del lenguaje matemático" (p.Il). Lo que demuestra la existencia del trabajo cooperativo y el desarrollo lógico matemático, con el dominio de extracción de datos, análisis, resolución, comprobación.

Según los investigadores Medina, L, Ruano, R. \& Caicedo, L. (2017) manifiestan que "La complejidad del conocimiento matemático se basa en la noción matemática en situación dual que dinamiza el proceso de enseñanza - aprendizaje" (p.256). 
Para Ayllón, F., Gómez, A., \& Ballesta-Claver, J. (2016), “el pensamiento matemático propicia el desarrollo de la creatividad debido a que requiere hacer conjeturas y discernir opciones que permitan resolver una situación planteada" (p.173).

En tanto que, Trigo, L. M. S. (2008) considera a la resolución de problemas matemáticos como la aplicación de un pensamiento en el cual los actores educativos dan solución a situaciones y las justifican con diferentes argumentos. Además, manifiesta que, el empleo de software educativo en la resolución de ejercicios y problemas matemáticos no solamente facilitan los procesos y estrategias, sino que fortalecen el proceso aprendizaje, de igual manera el uso de las herramientas tecnológicas, aportan a la conceptualización e interpretación de los problemas, como consecuencia permite que los estudiantes obtengan el desarrollo de sus habilidades.

Según García (1998) considera que "la resolución de problemas matemáticos es la base del aprendizaje, así también para adquirir conocimientos" (p. 146), deduciendo que la resolución de problemas es fundamental para cimentar el conocimiento y para alcanzar logros en la formulación, descubrimiento y planteamientos de problemas. Para Arreguín, E., Alfaro, A., \& Ramírez, S. (20I2) sostienen que "en los procesos de pensamiento se puede fomentar la competencia matemática para permitir entender situaciones problemáticas y aplicar informaciones a una gran variedad de situaciones y contextos" (p.2).

\section{MATERIALES Y MÉTODOS}

La investigación es cualitativa de acción-reflexión con enfoque cuantitativo y cualitativo, con el uso de métodos científicos. Para el estudio se tomó una población de 350 estudiantes, 10 docentes, con una muestra de 140 estudiantes de segundo curso y 105 estudiantes de tercero del Bachillerato General Unificado BGU de la Unidad Educativa Fiscal Margarita Cortes, de la ciudad de Esmeraldas, en el periodo lectivo 2021 - 2022. La investigación tuvo dos etapas. La primera, con un plan de investigación, donde se visibiliza el problema y objeto de estudio; a través de un sondeo participativo con docentes de matemática en el nivel de BGU del plantel educativo, panicularmente es la inducción a los docentes sobre el uso de software matemático en el proceso de enseñanza aprendizaje de quienes se recolecta datos sobre el nivel de uso del software matemático en la comprobación de solución de los ejercicios, mediante una encuesta. La segunda etapa, se realizó una intervención educativa proyectas a dos actividades, se fundamentó en la capacitación de 40 horas pedagógicas con 12 clases a los estudiantes del BGU de la Unidad Educativa Margarita Cortes, sobre el uso de software matemático en la comprobación de solución de los ejercicios, obteniendo datos sobre los beneficios del software a través de rubricas

La investigación y la intervención educativa contó con un comité ético; integrado por docentes de Matemática de altas experiencia, universitarios y de los planes educativos de bachillerato, quienes validaron los instrumentos de recolección de datos y realizaron sugerencias de orden semántico estructural, además de hacer el acompañamiento al 
proceso, toda vez que los resultados responde al proyecto de vinculación de la Facultad de la Pedagogía.

\section{RESULTADOS}

En entrevista con los docentes de Matemática del BGU de la Unidad Educativa Fiscal Margarita Cortes y tutores de vinculación de la Carrera de Pedagogía de las Ciencias Experimentales de las Matemáticas y Física de la Facultad de Pedagogía de la Universidad Técnica Luis Vargas Torres de Esmeraldas, se procesa los datos, según tabla I.

Tabla I. Resumen de procesamiento de casos

\begin{tabular}{|c|c|c|c|}
\hline \multirow{3}{*}{ Casos } & & $\mathrm{N}$ & $\%$ \\
\hline & Válido & 10 & 100,0 \\
\hline & Excluido $^{a}$ & 0 & ,0 \\
\hline & Total & 10 & 100,0 \\
\hline
\end{tabular}

a. La eliminación por lista se basa en todas las variables del procedimiento.

Fuente: Programa SPSS - Elaborado: Grupo Investigador

La prueba de Alfa de Cronbach revela el $97,80 \%$ de fiabilidad; por lo tanto, cumple con los estándares generalmente requeridos para este tipo de estudio socioeducativo.

Tabla 2. Estadísticas de fiabilidad

\begin{tabular}{cc}
$\begin{array}{c}\text { Alfa de } \\
\text { Cronbach }\end{array}$ & \multicolumn{1}{c}{$\begin{array}{l}\text { N de elementos } \\
\text { 978 }\end{array}$} \\
& $\begin{array}{l}\text { Fuente: Programa SPSS. } \\
\text { Elaborado: Grupo Investigador }\end{array}$
\end{tabular}

- Escaso uso de softwares matemáticos en la estrategiade $\quad 95 \%$ enseñanza - aprendizaje.

- Falta de inserción de softwares matemáticos como $90 \%$ recursos didácticos - tecnológicos

- Limitaciones de acceso a las aplicaciones y $98 \%$ herramientas tecnológicas

- Restricciones en la disponibilidad de uso de equipos $\quad 100 \%$ tecnológicos, en domicilio de los estudiantes.

- Deficiencia en la conexión a internet $86 \%$

Fuente: Profesores de la Unidad Educativa 
Margarita CortesElaborado: Grupo de investigación

Del diagnóstico de la investigación se estableció la necesidad de realizar una intervención educativa con la participación de estudiantes de la universidad y docentes de la unidad educativa. A los cuales se les capacitó sobre temas de software educativo, software matemático y tipos de aplicaciones para encontrar soluciones a ejercicios matemáticos, relacionados a los contenidos de segundo y terceros cursos del nivel de bachillerato.

En el plan de intervención se planteó la hipótesis: El uso del software matemático en la fase de aplicación del ciclo de aprendizaje ERCA promueve la autonomía de los estudiantes en la comprobación de la solución de ejercicios matemáticos. Para la demostración de la hipótesis se receptó dos cuestionarios tipo encuesta (pretest postest) y aplicando la prueba de muestras emparejadas se obtiene el siguiente resultado.

Tabla 3. Prueba de muestras emparejadas

\begin{tabular}{|c|c|c|c|c|c|c|c|c|c|}
\hline & & \multicolumn{5}{|c|}{ Diferencias emparejadas } & \multirow[t]{3}{*}{$\mathrm{t}$} & \multirow[t]{3}{*}{$g \mid$} & \multirow{3}{*}{$\begin{array}{l}\text { Sig. } \\
\text { (bilateral } \\
\text { ) }\end{array}$} \\
\hline & & \multirow[b]{2}{*}{ Media } & \multirow{2}{*}{$\begin{array}{l}\text { Desviació } \\
\text { n } \\
\text { estándar }\end{array}$} & \multirow{2}{*}{$\begin{array}{l}\text { Media de } \\
\text { error } \\
\text { estándar }\end{array}$} & \multicolumn{2}{|c|}{$\begin{array}{c}95 \% \text { de intervalo de } \\
\text { confianza de la diferencia }\end{array}$} & & & \\
\hline & & & & & Inferior & Superior & & & \\
\hline $\begin{array}{l}\text { Par } \\
\text { I }\end{array}$ & $\begin{array}{l}\text { Pretest } \\
\text {-Postest }\end{array}$ & $-3,40625$ & ,71208 & , 12588 & $-3,66298$ & $-3,14952$ & $-27,060$ & 31 &, 000 \\
\hline
\end{tabular}

Fuente: Elaboración propia.

El gl (grado de libertad) es el número de $\mathrm{N}$-I (la muestra menos I). Sig. (bilateral) o pvalor; éste indica la significancia de esas diferencias encontradas; para la decisión se considera el valor Alfa propuesto de 0,050; en este caso: p-valor: 0,000; Valor Alfa: $95 \%$ es decir que $5 \%$ de posibilidad de error que se convierte en 0,050 .

El resultado de la prueba $T$ de series relacionadas (pretest y postest) es $0.000<0.050$; por lotanto, se afirma que si existen relación significativa entre los resultados de pretest y postest; se demuestra que el uso del software matemático en la fase de aplicación del ciclo de aprendizaje ERCA favorece a la autonomía de los estudiantes en la comprobación de la solución de ejercicios matemáticos

\section{DISCUSIÓN}

Los resultados revelaron que los conocimientos los estudiantes sobre la comprobación de resultados de ejercicios mejoraron con uso de software matemático. En referencia al ciclo de aprendizaje se demostró que los estudiantes conocen las etapas de esta estrategia pedagógica, avalando los saberes de conceptos, símbolos, conceptos, teoremas. En cuanto a la fase de aplicación, se observó un alto porcentaje de manejo algorítmico, pero, en bajo porcentaje estaba seguro de obtener correctamente la 
respuesta en la resolución de ejercicios. Pero al utilizar un software para validar la respuesta se observa un cambio de conducta de los estudiantes.

Lo expuesto indica que la integración del software matemático para la comprobación de resolución de ejercicios contribuye al trabajo intelectual de los estudiantes, coincidiendo con lo señalado por Oteiza y Silva (200I), quienes visualizan el uso del software matemático en el proceso de enseñanza como un medio que facilita al estudiante a procesar la información de la asignatura, concordantes a los señalados, el investigador Esteban (2002), manifiesta que el uso de los software matemático como herramientas didáctica - pedagógica promueve el conocimiento y brinda apoyo en la comprensión de conceptos matemáticos.

Las opiniones de los docentes de matemática del nivel del Bachillerato General Unificado del Ecuador, ratifican que el apoyarse en las competencias digitales o uso software matemático desarrolla las prácticas de habilidades y destrezas de analogías, generalizaciones y otras operaciones intelectuales, coinciden, además, que se requiere de mucha dedicación y esfuerzo.

En este contexto y armonizando con lo manifestado de los docentes; Martínez (1999), c dice que para el desarrollo de las habilidades metacognitivas es necesario la práctica habilidades cognoscitivas y metacognitivas. Complementando esta premisa los investigadores Cuicas, (1997) y Sánchez, (2002), manifiestan que el software matemático, vinculados a los aprendizajes significativos, son herramientas que ayudan al desarrollo del pensamiento.

Además, el ciclo de aprendizaje experiencial sostenido por David Kolb citado por San Román (2016), afirma que una clase aplicada con el ciclo de aprendizaje ERCA sus estudiantes se motivación y asimilan los conocimientos unos a través de la experiencia, otros con una reflexión y para otros con una conceptualización o una aplicación, acorde con el enfoque socio cultural sostenido por Vygotsky, o por un aprendizaje por descubrimiento según Bruner, o por un aprendizaje por estadios de acuerdo a Piaget, o por un aprendizaje significativo como dice Ausubel, o por las inteligencias múltiples como manifiesta Howard, hacen que el estudiante logre competencias en el área de matemática.

\section{REFERENCIAS}

Ángel, J, y Bautista, G. (200I). Didácticas de las matemáticas en enseñanza superior: La utilización de software especializado. Recuperado el I 2 de enero de 2005, de http:// www.uoc.edu/web/esp/art/uoc/0107030/mates.html

Arreguín, L. E., Alfaro, J. A., \& Ramírez, M. S. (20I2). Desarrollo de competencias matemáticas en secundaria usando la Técnica de Aprendizaje Orientado en Proyectos.REICE. Revista Electrónica Iberoamericana sobre Calidad, Eficacia y Cambio en Educación. 
Ayllón, M. F., Gómez, I. A., \& Ballesta-Claver, J. (2016). Pensamiento matemático y creatividad a través de la invención y resolución de problemas matemáticos. Propósitos y representaciones, 4(I), 169-218.

Balderas, A. (2002). Didácticas de las matemáticas en Internet. Comunidades educativas y ambientes virtuales: Situación actual y perspectiva. Recuperado el 21 de enero de 2005 , de

Blanco, L., Cárdenas, J., Caballero, A. (2015) La resolución de problemas de matemáticas enla formación inicial de profesores de primaria. Recuperado.

https://mascruex.unex.es/ebooks/sites/mascruex.unex.es.mascvuex.ebooks/fi les/file s/file/Matematicas_9788460697602.pdf.

Díaz, F, y Hernández, G. (2002). Estrategias docentes para un aprendizaje significativo: Una interpretación constructivista ( $2 a$ ed.). México, D.F.: McGraw-Hill Interamericana.

Fernández, F., Izquierdo, J., y Lima, S. (2000). Experiencias en la estructuración de clases de matemáticas empleando asistentes matemáticos y colección de tutoriales hipermediales. Recuperado el 10 de marzo de 2005, de http://www.c5.cl/ieinvestiga/actas/ribie2000/papers//06/

Fernández, I., Riveros, V., \& Montiel, G. (2017). Software educativo y las funciones matemáticas. Una estrategia de apropiación. Omnia, 23(I), 9-19.

García, J. J. G. (1998). La creatividad y la resolución de problemas como bases de un modelodidáctico alternativo. Revista educación y pedagogía, (2I), I45-173. http://informaticaeducativa.com/coloquios/mesas/tres/angel/didactica.html

Martínez, A. (1999). Computadores en el aula: Herramientas mentales para la activación del pensamiento significativo. Recuperado 2 de febrero de 2005, de http://www.revele.com.ve/pdf/agenda/vol6-n2/pag9l.pdf

Medina, M., Ruano, G, \& Caicedo, L. (2017). Algunas apreciaciones acerca del proceso enseñanza-aprendizaje de la matemática. Dominio de las Ciencias, 3(1), 254-269.

Oteiza, F., y Silva, J. (200I). Computadores y comunicaciones en el currículo matemático: Aplicaciones a la enseñanza secundaria. Recuperado el 10 de mayo

Queralt, T. (2000). Un enfoque constructivista en el aprendizaje de las matemáticas con las calculadoras gráficas. Recuperado el 12 de enero de 2005 , de 
http://www.ti.com/calc/latinoamerica/pdf/Enfoque.pdf

Rodríguez, M. A. (20I7). Aplicación de ERCA como estrategia metodológica para mejorar el nivel de logro de aprendizaje de matemática en estudiantes de la I.E. Mario Vargas Llosa de Potracancha, Pillco Marca 2017.

Sánchez, M. (2002). La investigación sobre el desarrollo y la enseñanza de las habilidades del pensamiento. Recuperado el 20 de febrero de 2005, de http://redie.ens.uabc.mx/vol4nol/contenido-amestoy.html

Torregrosa-Gironés, G., Delicado, M. J. H., Martínez, M. D. C. P., \& Ciscar, S. L. (2010). Concepciones del profesor sobre la prueba y software dinámico. Desarrollo en un entorno virtual de aprendizaje Conception of teachers about proofs and dynamic software. Revista de educación, 352, 379-404.

Trigo, L. M. S. (2008). La resolución de problemas matemáticos: avances y perspectivas en la construcción de una agenda de investigación y práctica. In Investigación en educación matemática XII (p. 8). Sociedad Española de Investigación en Educación Matemática, SEIEM. 